\title{
Wikis y el nuevo estudiante de lenguas extranjeras
}

\author{
Wikis and the New Foreign Language Learner \\ Ana Gimeno Sanz \\ Universidad Politécnica de Valencia \\ agimeno@upvnet.upv.es \\ Jesús García Laborda \\ Universidad de Alcalá de Henares \\ jgarcial@upvnet.upv.es
}

\section{Resumen}

Los entornos digitales cada vez son más comunes en la enseñanza de lenguas extranjeras, además de jugar un papel muy importante como organizadores de tareas, proveedores de input y como gestores de recursos para la enseñanza de idiomas. Permiten, asimismo, a los profesores organizar la interacción de los estudiantes y, al mismo tiempo, proporcionar un magnífico entorno para presentar distintos géneros informativos. Las wikis son hoy en día una de las redes sociales que cada vez se utilizan más para dar apoyo al aprendizaje cooperativo y para fomentar el aprendizaje autónomo. Están estrechamente relacionadas con las metodologías docentes que se basan en el desarrollo de un proyecto o en la resolución de problemas, prácticas docentes éstas en las que tanto alumnos como profesores contribuyen a la construcción del conocimiento y comparten experiencias. Las wikis también han contribuido a crear las llamadas "comunidades de práctica", a las que pueden acudir los estudiantes en contextos de autoaprendizaje o en aquellas situaciones donde se carece del apoyo de un tutor o profesor para encontrar apoyo y consejo. Añadido a esto, la proliferación de herramientas que fomentan la comunicación mediatizada por ordenador han multiplicado los métodos docentes que se aplican en el aula y fuera de ella y han tenido una influencia muy significativa en el surgimiento de un nuevo perfil de estudiante; es decir, aquél que está plenamente acostumbrado a utilizar estas herramientas para relacionarse socialmente con otras personas aunque no sea con fines educativos. Por último, mediante el uso de las wikis se ha desarrollado el concepto de "enseñanza entre pares" y "tutorización entre pares", especialmente en contextos de creación de textos escritos mediante la cooperación colectiva. Este hecho ha contribuido, asimismo, a que el estudiante tenga mayor conciencia de los procesos implícitos en la construcción y edición textual. Este capítulo abarcará las wikis en términos generales y las enmarcará con respecto a las teorías sobre el aprendizaje y a las prácticas docentes.

Palabras clave: wiki, aprendizaje de lenguas, metodologías docentes, aprendizaje cooperativo

\begin{abstract}
Digital environments are becoming increasingly used in foreign language teaching and no doubt they play a very important role as task organisers, input providers and language teaching management resources. They also enable teachers to organise the learners' interaction and provide a remarkable framework for presenting different modes of information. Wikis are one of the increasingly used social network devices to support cooperative learning tasks in the language curriculum. They are closely associated to project-based and problem-based language learning practices where students and tutors alike contribute to the construction of knowledge and share findings. Wikis have also recently played a major role in creating communities of practice where learners can turn to for support and, in the event of self-access learning, can make up for a lack of guidance and tutor-support. In addition, the proliferation of such computer mediated communication
\end{abstract}

Red U - Revista de Docencia Universitaria. Número Monográfico V. Número especial dedicado a WIKI y educación superior en España (II parte), en coedición con Revista de Educación a Distancia (RED). http://www.um.es/ead/Red_U/m5/ 
Red U - Revista de Docencia Universitaria. Número Monográfico V. Número especial dedicado a WIKI y educación superior en España (II parte), en coedición con Revista de Educación a Distancia (RED). http://www.um.es/ead/Red_U/m5/

\begin{abstract}
devices have multiplied the pedagogic approaches to language learning and teaching, and have had a major influence on the emergence of a new student profile; i.e. that of the digital native who is accustomed to using such tools for social yet non-educational purposes. Lastly, through the use of wikis, the concept of "peer-to-peer" learning and "peer-tutoring" in collaborative writing environments have also largely evolved by fostering processes that support reflexive thinking and an increasing awareness of the production processes involved in writing and text editing. This article will therefore focus on 2 main aspects: a) A brief description of the main features that characterise wikis; and b) A tentative analysis of their effects on foreign and second language learning practices.
\end{abstract}

Keywords: wiki, language learning, teaching methods, cooperative learning

\title{
Introducción
}

Mucho ha cambiado la red desde la llegada en 1994 de la enciclopedia electrónica conocida como Wikipedia de la mano de Ward Cunningham ${ }^{\mathrm{i}}$. Ya sea con sus ventajas o sus inconvenientes -sobre los que se puede leer en esta monografía-, Wikipedia se ha convertido en una de las fuentes principales de información en la red a pesar de los peligros de plagio o uso no controlado de wikis con fines académicos (Schrock, 2007) y educativos (Engstrom y Jewett, 2005). Lo mismo ha sucedido, en menor medida, con wikis menos ambiciosas como aquéllas realizadas por comunidades escolares, alumnos de una asignatura, etc. Indudablemente, también el perfil del estudiante ha cambiado, al menos, en su relación con la red y las tecnologías de la información y de las comunicaciones.

En este capítulo nos centraremos en la evolución positiva sufrida por el discente de lenguas extranjeras en contacto con wikis (Lund, 2008a; Zorko, 2007). Prestaremos especial atención a los cambios en torno a tres aspectos fundamentales: responsabilidad del nuevo perfil de estudiante, necesidad del trabajo en equipo y un, creemos, renovado interés por el aprendizaje. No intentamos aquí, sin embargo, establecer una teoría del conocimiento sino, de una manera sencilla y tentativa -como corresponde a una primera aproximación a este tema-, establecer una serie de parámetros que describan con mayor o menor precisión este nuevo perfil de estudiante.

\section{Características sociales de las wikis}

Con la difusión de Internet, la red ha ido ofreciendo herramientas que fomentan la interactividad, el aprendizaje cooperativo, la adquisición de conocimientos de manera dinámica, etc. Este nuevo tipo de conocimiento se ve más abierto gracias a aplicaciones, programas y páginas web que no requieren grandes conocimientos técnicos informáticos y que facilitan la integración de contenidos de manera abierta y democrática. Por tanto, la participación se ha vuelto equitativa debido a la flexibilización de roles entre los administradores, aquéllos que organizan o desarrollan la aplicación o la página web, los gestores, quienes integran los contenidos, y los lectores o usuarios. Es a partir también del reparto de responsabilidades en la creación de contenidos -hecho que antes recaía únicamente en el administrador y que ahora, sin embargo, comparten todos los participantes en una web-, lo que conlleva y exige la creación de una conciencia respetuosa y compartida. Esta conciencia es, por naturaleza, crítica tanto en el formato de las wikis como, especialmente, en sus contenidos, que tienen que ajustarse a lo que sus usuarios desean genuinamente. Por ejemplo, en Wikipedia existe un gran esmero 
Red U - Revista de Docencia Universitaria. Número Monográfico V. Número especial dedicado a WIKI y educación superior en España (II parte), en coedición con Revista de Educación a Distancia (RED). http://www.um.es/ead/Red_U/m5/

Figura 1: Wiki del Colegio San Agustín de Valladolid. ${ }^{\text {ii }}$

Un ejemplo de wiki que pone de manifiesto un claro esfuerzo colectivo por alcanzar un objetivo común es la que creó el Aula de Teatro en Inglésiii de la Universidad Politécnica de Valencia (ver Figura 2). La finalidad de esta wiki era la traducción al inglés y la adaptación de la obra de Molière, El médico a palos, para su representación a final de curso. El profesor creó la wiki y, tras haber decidido sobre el reparto, pidió al grupo que tradujera el texto y lo adaptara a las necesidades del montaje. El proceso dio como fruto que cada actor y actriz tomara especial interés en su personaje y cuidara fundamentalmente su discurso, dotándolo incluso de rasgos personales. La motivación de esta experiencia estaba clara: fijar un texto para su representación por un grupo de teatro estudiantil en unos plazos muy determinados. El tiempo con el que se cuenta para llevar a cabo una experiencia de este tipo también es un factor determinante para su éxito. El texto debía estar listo para los ensayos. Sin embargo, fue precisamente la premura de tiempo la que hizo que los ensayos empezaran antes de que estuviera adaptado por completo el texto, por lo que los dos procesos coexistieron (el de la adaptación y el de los ensayos de la obra), convirtiendo, así, la experiencia en un constante proceso orgánico de escritura y revisión.

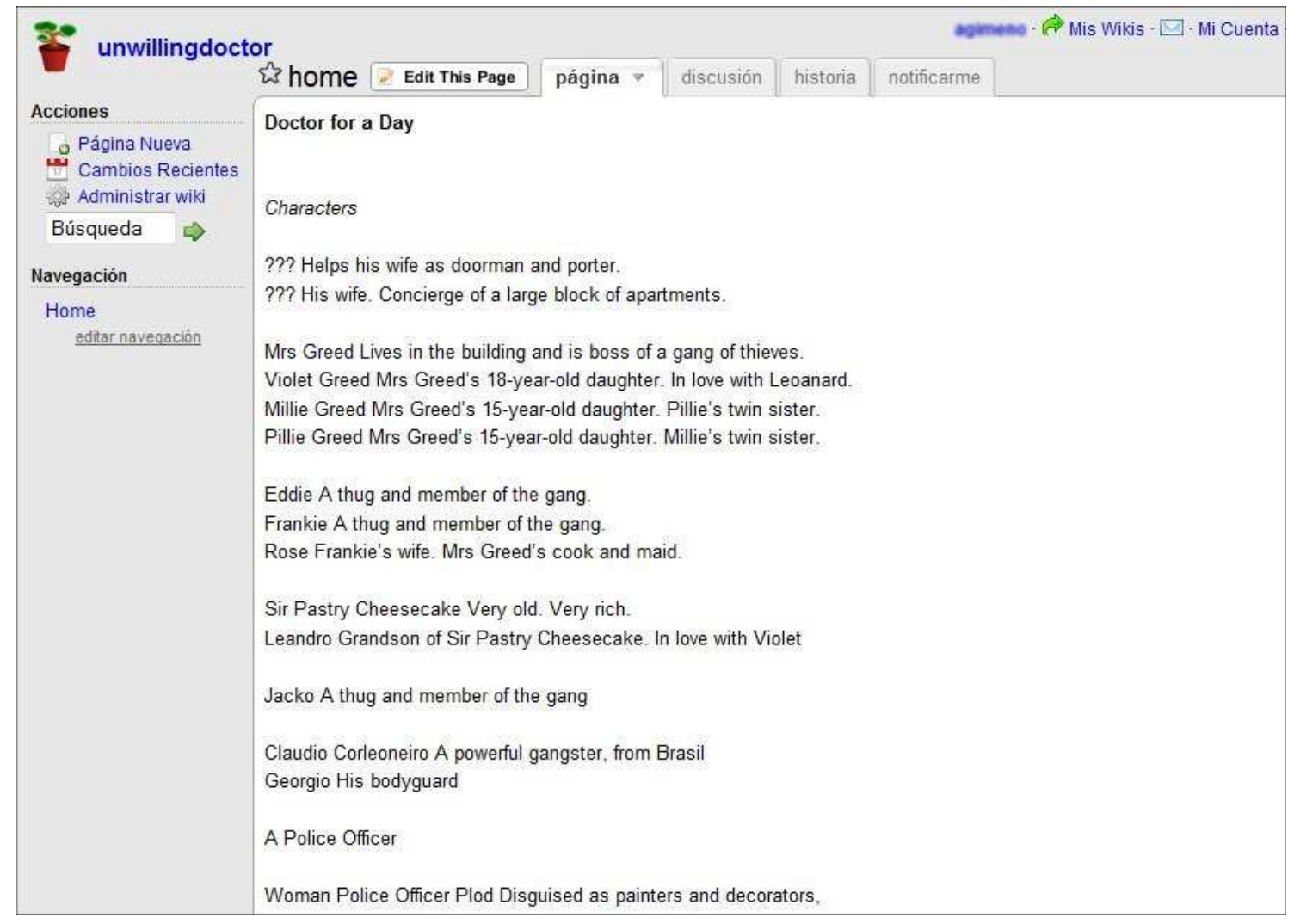

Figura 2: Wiki del aula de Teatro en Inglés de la Universidad Politécnica de Valencia para traducir y adaptar la obra de Molière, El médico a palos.

Creemos que éste es un buen ejemplo de tres de las características esenciales de una wiki puesta al servicio de un fin educativo: que exista una motivación que suscite la wiki, que la finalidad esté bien delimitada y que los contenidos se creen en un tiempo delimitado. La dilatación en el tiempo podría, pensamos, disuadir a muchos estudiantes en el cumplimiento del cometido. 
Red U - Revista de Docencia Universitaria. Número Monográfico V. Número especial dedicado a WIKI y educación superior en España (II parte), en coedición con Revista de Educación a Distancia (RED). http://www.um.es/ead/Red_U/m5/

Pese a que la función primordial de una wiki es proporcionar una información determinada a una comunidad mayor o menor de usuarios, uno de los usos más enriquecedores es precisamente el apartado de "discusión" o "comentarios" que incluye toda wiki por defecto. Ahí es, por lo tanto, el lugar en el que se puede producir la interacción entre usuarios, ya que propicia el que cada usuario tenga la oportunidad de defender una postura, idea o creencia respecto al contenido de una página.

Como en toda relación social, hay agentes (los usuarios de la wiki) activos y pasivos. En lo que respecta a los procesos educativos, aunque el hecho de ser lector pasivo de los contenidos de una wiki puede considerarse como parte legítima del aprendizaje, sólo aporta a éstos la posibilidad de asimilar, recordar y reproducir esa información. Por el contrario, para conseguir objetivos de otro orden, tales como el pensamiento crítico o dotes persuasivas, la participación activa se hace absolutamente necesaria. Será precisamente esa interacción con otros usuarios la que favorecerá el desarrollo de esas aptitudes intelectuales.

\section{Hacia una teoría del conocimiento}

En los últimos años se viene planteando la complementariedad de varios estilos cognitivos en la formación de los discentes: un aprendizaje más abstracto y uno más experiencial. Mientras que el primero se asocia a los contenidos más teóricos obtenidos a través de la lectura o la formación, el segundo se asocia a la necesidad de que aquello que se ha aprendido de manera teórica se refuerce mediante su experimentación. Los conocimientos transmitidos a través de páginas web y wikis se asocian con el primero (Forte y Bruckman, 2007). Las teorías de Lev Vygotsky (citado por Galloway, 2001), obviamente relacionadas con este aprendizaje abstracto, han adquirido una fuerza considerable en los últimos años, ya que en casos como las wikis, éstas actúan como intermediarias (como una interfaz) entre el conocimiento ya existente y el adquirido por el alumno. Sin embargo, para Saymour Papert (citado por Kim, 2001) es necesario que ese conocimiento se convierta en un producto acabado para que se realice un aprendizaje completo. Al contrario que en el aprendizaje experiencial, el constructivismo social de Saymour Papert requiere un conocimiento propio para ser internalizado. Por ejemplo, un alumno internalizaría y asimilaría mejor el estilo indirecto en inglés si se le pide escribir una entrada para la wiki de la clase en la que ponga en práctica este elemento gramatical. Por tanto, los profesores y los estudiantes de lenguas extranjeras incrementan o afianzan sus conocimientos a través de la creación de entradas y artículos en wikis que posteriormente van a ser utilizadas por sus propios alumnos o compañeros (Figura 3 ). 
Red U - Revista de Docencia Universitaria. Número Monográfico V. Número especial dedicado a WIKI y educación superior en España (II parte), en coedición con Revista de Educación a Distancia (RED). http://www.um.es/ead/Red_U/m5/

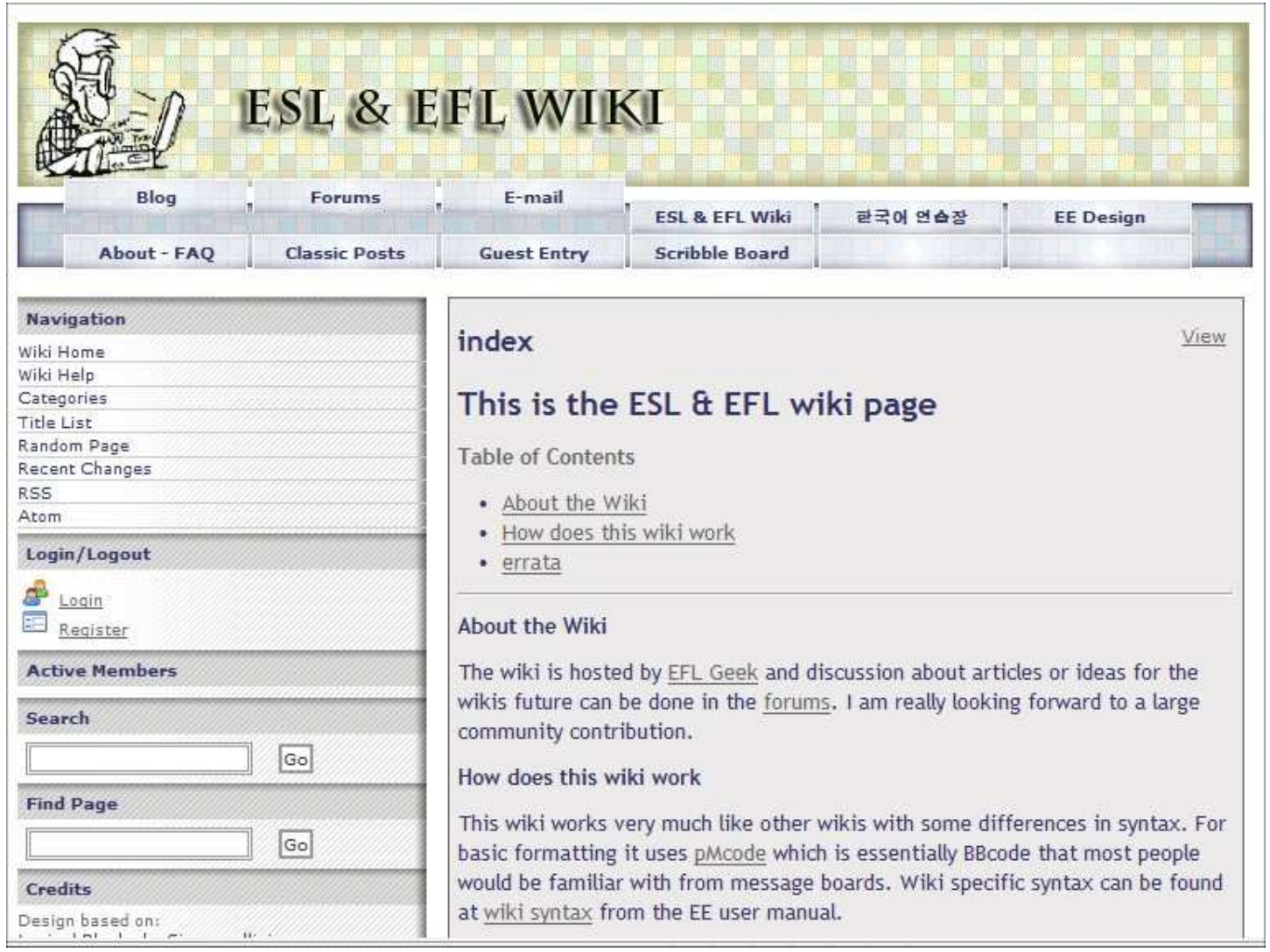

Figura 3: Ejemplo de wiki para la enseñanza de inglés como segunda lengua. ${ }^{\text {iv }}$

Además de estas circunstancias, el constructivismo social incluye otra serie de características que definen a los alumnos:

a. Los alumnos aprenden los unos de los otros. Es decir, el profesor puede delegar aspectos de la lengua, la literatura o la cultura para que los alumnos se sientan corresponsables en el proceso didáctico e incluyan sus entradas de manera positiva, fidedigna y equilibrada en la wiki. Además, los alumnos deben sentirse capaces de proponer modificaciones, aclaraciones y añadir nueva información.

b. Responsabilidad en la distribución del contenido. Los alumnos deben ser responsables de la veracidad y la corrección de los contenidos para no difundir conocimientos que guíen a sus compañeros de manera errónea.

c. Interactividad. Los estudiantes participan de manera activa y continuada junto con sus compañeros.

d. La cercanía de la escritura y la publicación del trabajo. En ocasiones hay temas que no se ven convenientemente reflejados en ninguna biblioteca o publicación; a veces por razones de la vaguedad de los contenidos. Sin embargo, puede darse el hecho de que el público o el estudiante tengan interés por esos temas. Por tanto, los alumnos sienten la invitación a participar en el desarrollo de los contenidos sabiendo que se les tiene en consideración y que, en algunos casos, hasta recibirán retroalimentación de su participación ya sea sobre el contenido o en la forma de construir la wiki (Forte y Bruckman, 2006). ${ }^{\mathrm{v}}$

e. Necesidad del tutor o moderador. Ésta ha sido una necesidad señalada en numerosas wikis. En muchas wikis escolares se crean mecanismos de control para que la información introducida por los estudiantes sea veraz y objetiva. 
Red U - Revista de Docencia Universitaria. Número Monográfico V. Número especial dedicado a WIKI y educación superior en España (II parte), en coedición con Revista de Educación a Distancia (RED). http://www.um.es/ead/Red_U/m5/

f. El aprendizaje cognitivo. Lo fundamental es el conocimiento frente a la experiencia. En este sentido, la transmisión de información es un aspecto fundamental de las wikis. De alguna manera, el conocimiento procedimental que desbancó al cognitivo en los años 90 se convierte en apoyo o una vía secundaria de aprendizaje. En este tipo de aprendizaje el método de casos adquiere una gran importancia.

g. Aprendizaje cooperativo. Los alumnos son corresponsables del aprendizaje propio y de sus compañeros.

Una de las claves del éxito de las wikis en entornos docentes consiste precisamente en su carácter eminentemente cooperativo. Es, creemos, ese carácter público de la producción escrita y el hecho de que el estudiante puede contribuir a abrir una nueva ventana al mundo lo que les resulta atractivo y motivador a los estudiantes. A través de esa colaboración online los alumnos tienen acceso a una fuente de información tremendamente valiosa que en entornos docentes más tradicionales es difícil de conseguir. Hemos comprobado que, cuando un estudiante se embarca en un proyecto de creación de una wiki con una finalidad específica y bien delimitada, supone un reto personal intentar mejorar la producción escrita de los compañeros, por lo que se esfuerzan en producir contenidos "merecedores" de formar parte de ese escaparate público. Por otra parte, también es cierto que se multiplican las posibilidades de interactuar con otros estudiantes de lenguas ofreciendo, por tanto, más oportunidades de practicar la lengua objeto de aprendizaje. Son diversas, naturalmente, las situaciones en las que se puede producir la colaboración entre pares en tareas escritas, pero lo que es indudable es la necesidad de que las tareas encomendadas estén bien diseñadas y tengan un cometido y un objetivo concretos con los que el estudiante se sienta cómodo y que pueda hacer suyos. Las actividades didácticas que conllevan tareas con wikis pueden producirse tanto en entornos de aprendizaje reglado, como en situaciones de aprendizaje informal. Esto, naturalmente, afectará también la orientación de las tareas. Otro factor determinante es que las wikis han permitido transformar el concepto tradicional de trabajo en grupo dentro del aula (realización de tareas en grupo), convirtiéndolo en una actividad no presencial, de carácter autónomo, que ofrece la posibilidad de interactuar con comunidades cuyas dimensiones pueden oscilar desde grupos reducidos a números ilimitados de agentes.

Cabría recordar, sin embargo, la diferencia entre tareas en las que los estudiantes colaboraran los unos con los otros (aprendizaje colaborativo) y aquéllas en las que cooperan entre ellos (aprendizaje cooperativo). La siguiente tabla ${ }^{\mathrm{vi}}$ resume, creemos, adecuadamente la diferencia entre ambas:

\section{Cooperative Learning}

Definition: Cooperative learning is a successful teaching strategy in which small teams, each with students of different levels of ability, use a variety of learning activities to improve their understanding of a subject. Each member of a team is responsible not only for learning

\section{Collaborative Learning}

Definition: "Collaborative learning is based on the idea that learning is a naturally social act in which the participants talk among themselves (Gerlach, 1994). It is through the talk that learning occurs." 
Red U - Revista de Docencia Universitaria. Número Monográfico V. Número especial dedicado a WIKI y educación superior en España (II parte), en coedición con Revista de Educación a Distancia (RED). http://www.um.es/ead/Red_U/m5/

\begin{tabular}{|c|c|}
\hline $\begin{array}{l}\text { each person is responsible for a } \\
\text { portion of the work }\end{array}$ & $\begin{array}{l}\text { participants work together to solve a } \\
\text { problem }\end{array}$ \\
\hline $\begin{array}{l}\text { many times the teacher already } \\
\text { knows the problem and solution } \\
\text { students will be working towards }\end{array}$ & $\begin{array}{l}\text { many times teacher does not have a } \\
\text { pre-set notion of the problem or } \\
\text { solution that students will be } \\
\text { researching }\end{array}$ \\
\hline
\end{tabular}

Como podemos ver, las tareas docentes con wikis caen dentro de la categoría de aprendizaje cooperativo en el que cada aprendiz contribuye con su aportación para el bien colectivo, a diferencia de lo que sucedería en el de carácter colaborativo, en el que es el conjunto el que colectivamente avanza y progresa para alcanzar el objetivo común. Sin embargo, también puede darse el caso de que un proyecto comience de forma cooperativa y acabe siendo una tarea colaborativa a medida que se van planteando más preguntas, relaciones e ideas.

\section{¿Un aprendizaje cognitivo o social constructivista?}

Una de las cuestiones principales, como hemos dicho en otros puntos de este artículo, es dónde recae la responsabilidad y el origen del conocimiento (Royal y Kapila, 2009). En nuestra opinión, el aprendiz es el punto central del aprendizaje. Efectivamente, tanto el profesor como las tareas correspondientes a la creación de la wiki tienen un valor fundamental y todo ello está mediado por el contexto (en este caso, electrónico). Sin embargo, aunque muchos tienden a sobrevalorar el aspecto contextual, es necesario concederle un valor relativo no mayor que los otros. Como veremos posteriormente, la creación de wikis tiene una responsabilidad y un impacto social que han sido poco estudiados desde un punto de vista educativo, aunque sociológicamente se pueden definir como la conciencia de estar escribiendo en un medio abierto y accesible por una comunidad de mayor o menor dimensiones (desde una clase a una wiki abierta en Internet), por lo que valores como veracidad y objetividad, así como el compromiso con el público potencial, introducen una responsabilidad compartida tanto por el docente como por el discente. Por tanto, el alumno tiene dos responsabilidades; por una parte, como receptor (de la información transmitida por su profesor y de las fuentes de información de las que adquiere el conocimiento que luego procesará) y, por otra, como transmisor. Pero estas dos funciones se encuentran mediadas por procesos cognitivos de comprensión, interpretación, procesamiento lógico y expresión. Este proceso es totalmente cognitivo y aunque el constructivismo social ha hecho un esfuerzo por no mostrar claramente estos pasos, no dejan de ser necesarios. Por tanto, la perspectiva socioconstructivista requiere una revisión de tres pasos orientados por el profesor: procesamiento de contenidos, diseño de los procedimientos de trabajo y planeamiento meticuloso de de las tareas. De esos procesos resulta inevitable reflejar las actitudes, valores y motivaciones de los profesores que, por supuesto, son inherentes a los tres aspectos. Por ejemplo, un profesor que considera importante realizar una wiki 
Red U - Revista de Docencia Universitaria. Número Monográfico V. Número especial dedicado a WIKI y educación superior en España (II parte), en coedición con Revista de Educación a Distancia (RED). http://www.um.es/ead/Red_U/m5/

sobre costumbres del Reino Unido ha pensado en esos valores positivos que se quiere explotar, selecciona los materiales que subjetivamente cree que beneficiarán al alumno y orienta la expresión de los mismos. Por tanto, la creación de las wikis se convierte en la interfaz integral (cognitiva, sociocognitiva e ideológica) entre los profesores y los alumnos. Por consiguiente, la creación de la wiki es producto de una negociación entre el estudiante, el profesor, el entorno y el receptor (no hablamos aquí únicamente de lector ya que podría tener acceso a archivos de sonido), que puede ser constatada en los contenidos, la forma de presentarlos, la redacción, las correcciones y la forma de editar la producción del alumno -por el profesor u otros estudiantes- (Morgan y Smith, 2008; Toker, Mosely y Chow, 2008).

\section{Metodologías docentes}

En tanto que sistema de composición de textos, medio de comunicación asíncrono y repositorio de contenidos, las wikis son, ante todo, una herramienta para la colaboración entre usuarios (en nuestro caso, estudiantes de lenguas extranjeras). Y es precisamente esta característica la que determina en gran medida las metodologías docentes a emplear. Algunos de los métodos más apropiados, en nuestra opinión, son el aprendizaje basado en la resolución de problemas y el que se basa en el desarrollo de un proyecto preestablecido. Este último se enmarca dentro del modelo constructivista y se basa en los principios de la producción textual por parte del estudiante. Se sustenta en la búsqueda fundamentada de información con la finalidad de involucrar al estudiante en los procesos de aprendizaje profundo ${ }^{\text {vii }}$ a través del pensamiento crítico y analítico de las ideas y de la información recibida para relacionar éstas con los conocimientos previos y provocar, así, la comprensión y retención duradera de los conocimientos (recién) adquiridos. La finalidad última de este enfoque es provocar en los estudiantes la necesidad de investigar por sí mismos para llegar a comprender y, por consiguiente, asimilar nuevos conceptos e ideas.

El aprendizaje basado en la resolución de problemas cada vez cobra mayor popularidad en la enseñanza universitaria para fomentar una participación activa en los procesos de adquisición de conocimientos. Las características que lo definen, muy someramente, son, por una parte, la creencia de que se puede estimular el aprendizaje planteándoles a los estudiantes problemas abiertos, y, por otra, provocando cierto grado de responsabilidad para escoger los métodos y procedimientos para la resolución satisfactoria de los problemas. Se persigue, de esta manera, estimular a los alumnos para que trabajen en grupo ayudándose los unos a los otros, a la vez que el profesor se convierte en mero guía o "facilitador", en lugar del tradicional rol de transmisor de conocimiento. En este sentido, la capacidad de resolver problemas no se restringe a acumular conocimientos, sino que implica adquirir la capacidad de enfrentarse a situaciones desestructuradas para aportar una solución adecuada a un problema real (Jones 1996). También se cree que el aprendizaje basado en la resolución de problemas ayuda a fomentar la adquisición de conocimientos relacionados no sólo con la lengua objeto de estudio, sino relacionados también con la materia en la se encuadra el problema por resolver, además de ayudar a desarrollar dotes de comunicación y de autoaprendizaje.

En el contexto del aprendizaje integrado de contenido y lenguas (AICLE) ${ }^{\text {viii }}$ o en el de la enseñanza de lenguas para fines específicos, los problemas a plantear en el aula pueden, naturalmente, tomar una variedad considerable de formas dependiendo de la 
Red U - Revista de Docencia Universitaria. Número Monográfico V. Número especial dedicado a WIKI y educación superior en España (II parte), en coedición con Revista de Educación a Distancia (RED). http://www.um.es/ead/Red_U/m5/

materia con la que se combina el aprendizaje de la lengua extranjera. Este es un contexto claro, creemos, en el que las tecnologías de la información y de las comunicaciones en general, y muy particularmente las wikis, pueden jugar un papel importante para ofrecer el apoyo necesario tanto al profesor como a los estudiantes. Se pueden crear actividades basadas en la resolución de problemas relacionados con la mayoría de las asignaturas incluidas en los diversos planes de estudio, si bien es cierto que, para diseñar actividades que combinen la doble vertiente de servir de base para la enseñanza de la materia en cuestión y una lengua extranjera, requiere una planificación sumamente cuidadosa por parte de los especialistas (el/los de la materia y el/los de la lengua extranjera). Se ha de encontrar el equilibrio entre los dos enfoques, si bien el objetivo primordial sea adquirir conocimientos y habilidades relacionados con la materia objeto de estudio.

En lo que se refiere al desarrollo de un proyecto, debido a que esta metodología enfatiza el aspecto cooperativo del aprendizaje entre los propios estudiantes para llevar a buen término un proyecto determinado, los integrantes del grupo contarán con una cantidad variable de input derivado de las aportaciones de otros compañeros. Otra característica del aprendizaje que se basa en el desarrollo de un proyecto es que fomenta el aprendizaje autónomo, la capacidad de análisis y el aprendizaje a lo largo de toda la vida. Tal y como se expone en "EduTechWiki", ix una wiki destinada a la enseñanza creada en 2006 por Daniel Schneider:

The topics of autonomy and independence play an increasingly important role in language education. They raise issues such as learners' responsibility for their own learning, and their right to determine the direction of their own learning, the skills which can be learned and applied in self-directed learning and capacity for independent learning and the extents to which this can be suppressed by institutional education.

Como vemos, en el aprendizaje autónomo, el profesor ha de contar con el deseo por parte del estudiante de asumir un alto grado de responsabilidad sobre su proceso de aprendizaje y debe, por lo tanto, estar dispuesto a que ese proceso no transcurra según lo previsto. El alumno tendrá, por ende, un mayor grado de libertad a la hora de reconducir y tomar decisiones sobre el cómo, cuándo y dónde aprende.

En esta misma wiki se nos recuerda que según Henri Holec (1979), considerado "el padre del aprendizaje autónomo":

[...] the autonomous language learner takes responsibility for the totality of his learning situation. He does this by determining his own objectives, defining the contents to be learned and the progression of the course, selecting methods and techniques to be used, monitoring this procedure, and evaluating what he has acquired. Objectives are specific to the learner, and the learner's communicative needs determine the verbal elements chosen. Learning thus proceeds from ideas to correct grammatical, lexical, and phonological form. The self-directed learner chooses the methods of instruction through trial-and-error. His selection is based on the objectives set and its applicability to internal and external constraints. The student evaluates his attainment through his objectives, and this evaluation helps him to plan subsequent learning. The concept of autonomous learning requires a redefinition of knowledge from an objective universal to a subjective individual 
Red U - Revista de Docencia Universitaria. Número Monográfico V. Número especial dedicado a WIKI y educación superior en España (II parte), en coedición con Revista de Educación a Distancia (RED). http://www.um.es/ead/Red_U/m5/

knowledge determined by the learner. For teachers, it means new objectives which help the learner define his personal objectives and help him acquire autonomy.

Aunque en el desarrollo de un proyecto común se le transfiere al estudiante cierto grado de autonomía, no llega sin embargo, esta autonomía al grado del llamado aprendizaje independiente ya que para llevar a cabo la tarea encomendada (utilizando para ello el entorno de una wiki) el estudiante contará con el apoyo y la contribución de otros aprendices, además de la supervisión del profesor.

\section{Wikis y el aprendizaje de lengua extranjera}

Las wikis promueven el conocimiento propio. Tal y como se ha mencionado, académicamente se aprende mejor enseñando lo conocido a los demás. Por tanto, el hecho de que los documentos wiki sean resultado de una construcción común (ya sean guías, manuales, diccionarios, portafolios, proyectos culturales u otros) favorece la función dual alumno-profesor. Es decir, cuando un alumno tiene que introducir un ítem sobre un aspecto lingüístico o cultural de una lengua extranjera sigue el siguiente proceso:

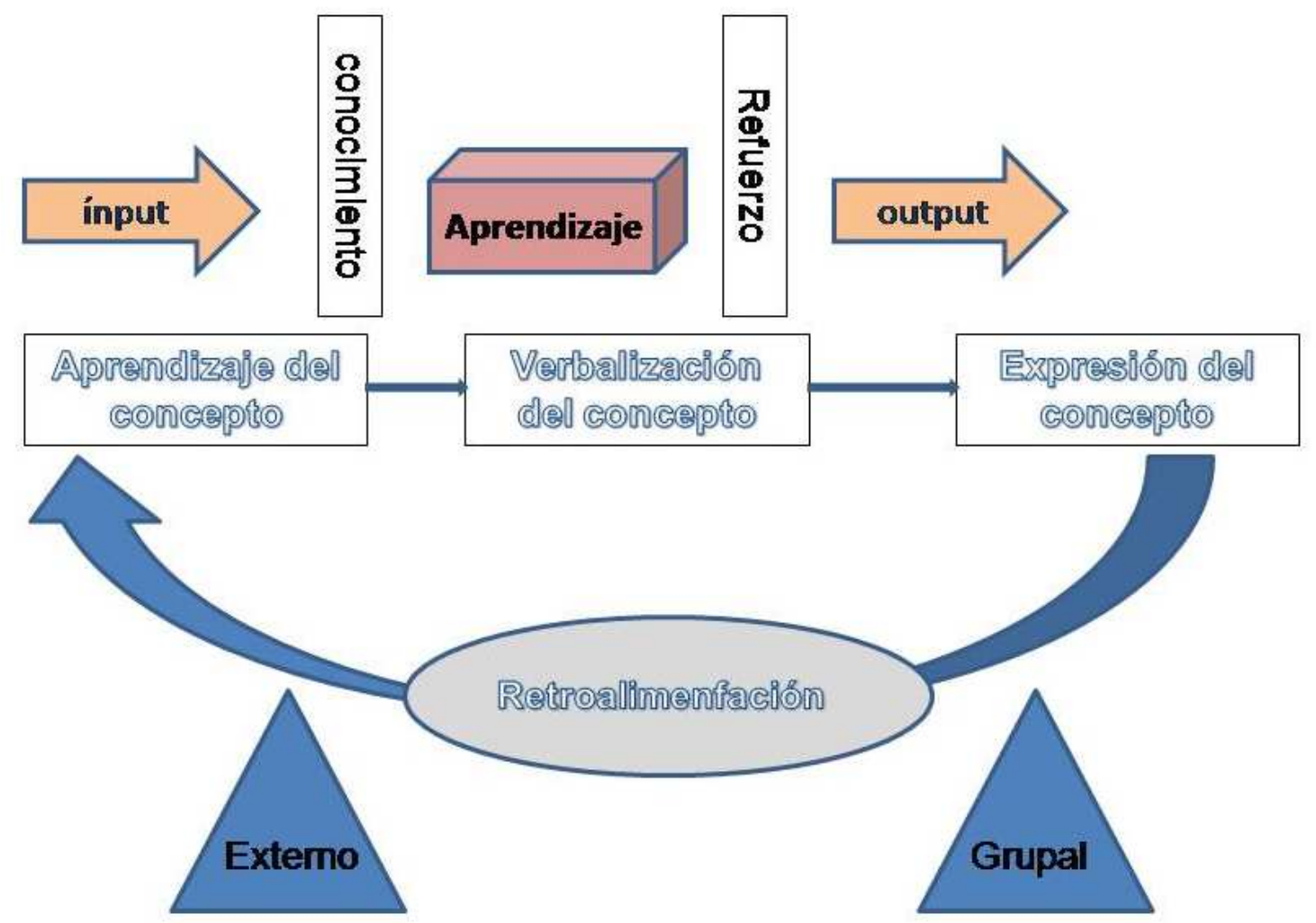

Figura 4: Proceso cognitivo del estudiante-participante en una wiki. (Davidson, 2008; Xiao y Lucking, 2008).

Tal y como queda reflejado en la Figura 4, en el proceso del desarrollo de la wiki participan tres procesos fundamentales: el input, el output y la retroalimentación especialmente esta última- (Davidson, 2008; Xiao y Lucking, 2008). Los tres son también fundamentales en el aprendizaje de lenguas extranjeras (Ellis, 1995; Krashen, 1988). Por tanto, el procesamiento mental del alumno sigue un patrón de aprendizaje 
Red U - Revista de Docencia Universitaria. Número Monográfico V. Número especial dedicado a WIKI y educación superior en España (II parte), en coedición con Revista de Educación a Distancia (RED). http://www.um.es/ead/Red_U/m5/

que, en el caso de wikis asociadas al aprendizaje de lenguas extranjeras, es fundamental. Asimismo, la introducción y uso de los programas informáticos permiten también el desarrollo de las competencias tecnológicas tan deseadas en el ámbito general y especialmente en el universitario (García Laborda, 2002), al tiempo que incrementan la motivación del alumno. Además, convendremos sobre la importancia de la retroalimentación en la segunda lengua, tanto proveniente de los propios compañeros como de los conocimientos modificados adquiridos por fuentes externas (libros, profesor, etc.). Por consiguiente, las wikis deben estar (y generalmente están) pensadas para que sean accesibles tanto en su uso como en la posibilidad de incluir contenidos (textos, diagramas, resúmenes o imágenes) para un amplio espectro de usuarios, ya sean alumnos o profesores, con distintas habilidades informáticas que, en el marco estructural sencillo de manejo de una wiki, aporten sus noticias o conocimientos. Es decir, que aquellos contenidos que incluyen se conviertan en fuentes de input para el resto de la comunidad discente. Así pues, las wikis tienen la ventaja inherente de que su software no suele quedar desfasado y es flexible a la hora de poder incluir nuevos contenidos sin tener que quitar o eliminar los precedentes. Por eso, tanto docentes como discentes tienen la posibilidad de ver la evolución y el aprendizaje de un alumno determinado a través de su producción escrita. Asimismo, todos los participantes pueden aprender de las ideas aportadas por el mismo en un formato que es amistoso y fácilmente comprensible por todos los participantes.

De cualquier manera, y centrándonos en el aspecto de los contenidos, las wikis tienen un carácter muy pedagógico porque permiten reflejar la retroalimentación, que puede venir tanto del profesor (por ejemplo, en forma de correcciones de gramática, estilo, etc.) como de los alumnos (a través de datos o comentarios nuevos). Por eso, un aspecto fundamental en el uso de wikis y el aprendizaje de lenguas extranjeras es que se establece en entornos en los que la telecooperación internacional es real, como ocurre cuando entran en contacto estudiantes multilingües o de diversos orígenes. En este caso, la necesidad continuada de comunicación (Lund, 2008a) se establece a tres niveles (la wiki, la comunicación síncrona y la asíncrona) que promueven tres tipos de actividades de aprendizaje tal y como se muestra en las Figuras 5 y 6 , pero que, en conjunto, influyen muy especialmente el desarrollo de la lectoescritura (Mak y Coniam, 2008; Morgan y Smith, 2008) y de la competencia comunicativa del participante (Stacey, 2008).

\begin{tabular}{|c|c|c|}
\hline Tipo de comunicación & $\begin{array}{l}\text { Destrezas desarrolladas } \\
\text { por el estudiante de } \\
\text { segunda lengua }\end{array}$ & Efecto en el estudiante \\
\hline Wiki & Lectura y escritura & $\begin{array}{l}\text { Desarrollo de la capacidad } \\
\text { lecto-escritora, aprendizaje de } \\
\text { léxico, mejora en la habilidad } \\
\text { de la expresión escrita } \\
\text { (especialmente la académica). }\end{array}$ \\
\hline Síncrona & $\begin{array}{l}\text { Habla y escucha (primaria) } \\
\text { Lectura y escritura }\end{array}$ & $\begin{array}{l}\text { Comunicación dirigida a } \\
\text { proyectos comunes. } \\
\text { Comunicación especialmente } \\
\text { académica, pero conviene }\end{array}$ \\
\hline
\end{tabular}


Red U - Revista de Docencia Universitaria. Número Monográfico V. Número especial dedicado a WIKI y educación superior en España (II parte), en coedición con Revista de Educación a Distancia (RED). http://www.um.es/ead/Red_U/m5/

\begin{tabular}{|l|l|l|}
\hline & (secundaria) & $\begin{array}{l}\text { recordar que cuando hay una } \\
\text { comunicación entre } \\
\text { estudiantes es difícil limitarse } \\
\text { a lo estrictamente académico } \\
\text { (como sucede en el caso } \\
\text { anterior) por la propia } \\
\text { naturaleza del lenguaje, por lo } \\
\text { que este tipo de } \\
\text { comunicación es } \\
\text { extremadamente } \\
\text { enriquecedor. } \\
\text { Asíncrona }\end{array}$ \\
Lectura y escritura \\
$\begin{array}{l}\text { Este es un aspecto } \\
\text { generalmente informativo. } \\
\text { Aunque existen ciertos } \\
\text { aspectos de los dos anteriores, } \\
\text { en general prevalece ese } \\
\text { aspecto informativo o de } \\
\text { retroalimentación. }\end{array}$ \\
\hline
\end{tabular}

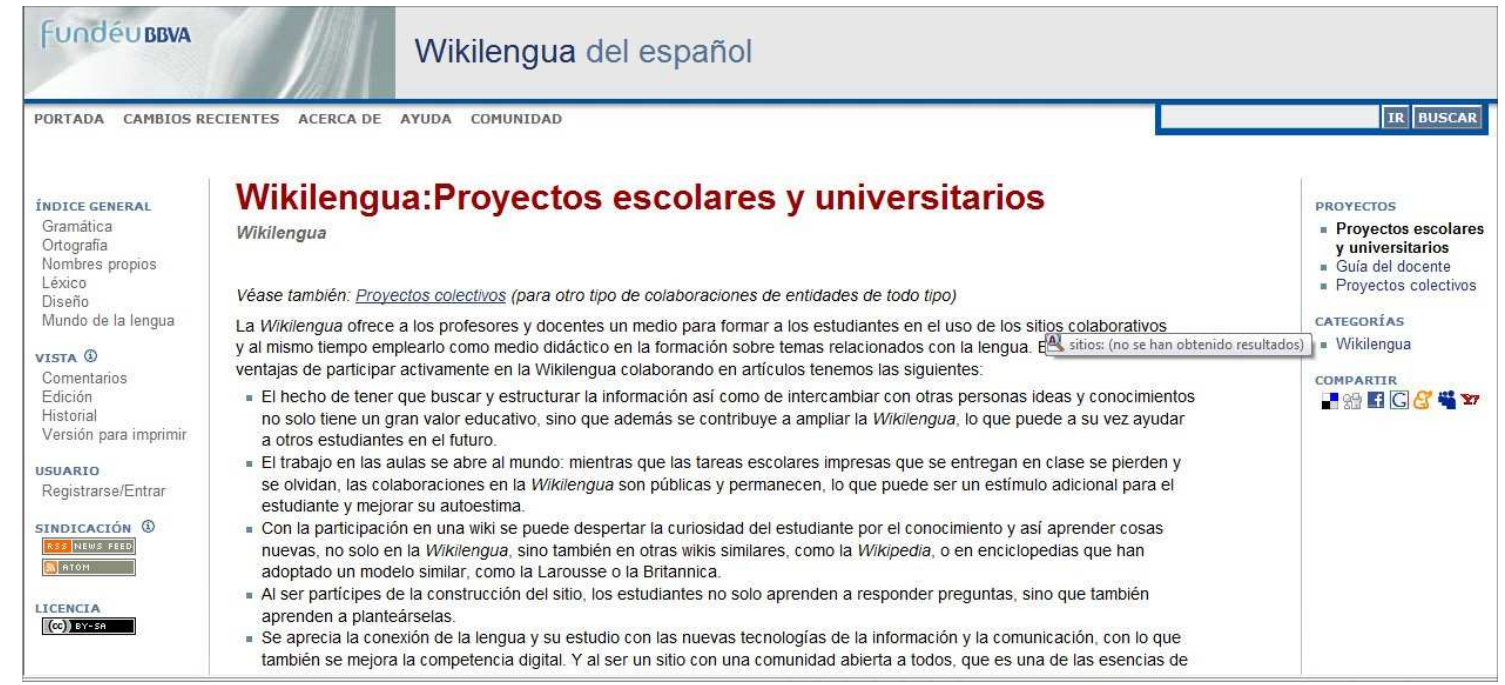

Figura 5: Participación de los distintos tipos de comunicación en proyectos cooperativos en distintas lenguas. 
Red U - Revista de Docencia Universitaria. Número Monográfico V. Número especial dedicado a WIKI y educación superior en España (II parte), en coedición con Revista de Educación a Distancia (RED). http://www.um.es/ead/Red_U/m5/

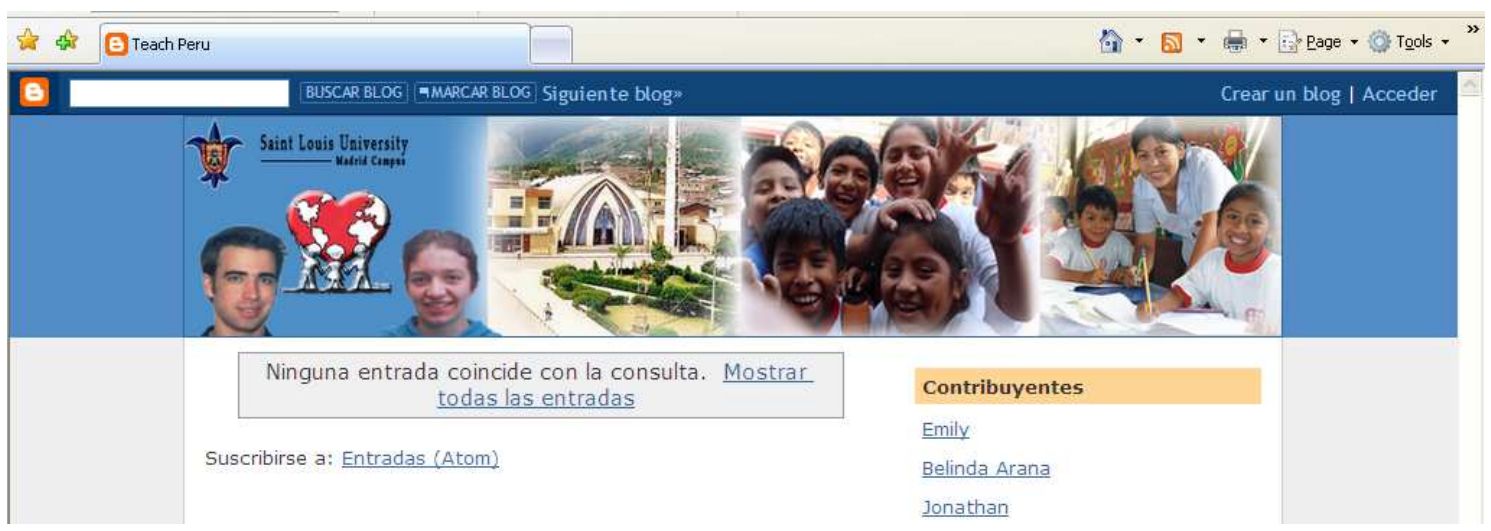

Figura 6: Proyecto cooperativo entre la Universidad de Saint Louis y Teach Peru.

El efecto de las wikis es, como hemos señalado, polifacético. El trabajo con wikis, como sucede con otras aplicaciones educativas informáticas, ha sido generalmente enfocado desde la perspectiva del constructivismo social, pero no podemos dejar de lado los aspectos cognitivos del proceso de creación, desarrollo y mantenimiento propios de la enseñanza de lenguas asistida por ordenador. Estos aspectos requieren la participación de procesos cognitivistas que paulatinamente parecen ser cada vez menos utilizados (García Laborda, 2009). Así pues, los factores incidentales que sin duda afectan al papel pedagógico de las wikis en materia de lenguas extranjeras son: el papel del profesor, las diferencias individuales, los efectos de la motivación, la incidencia en los procesos de aprendizaje, el lugar de las tareas en la creación de wikis y los efectos del contexto en el aprendizaje con wikis.

\section{Las wikis para los profesores}

De la misma manera que los estudiantes elaboran sus trabajos, los docentes pueden establecer wikis o secciones en las wikis para orientar a sus alumnos. Aunque en otros artículos de esta revista se muestran experiencias y métodos docentes cooperativos basados en el trabajo común, recordaremos algunas ventajas del trabajo cooperativo docente en materia de wikis.

a. Unificación de información. Los profesores no necesitan introducir sus datos varias veces y los tutoriales pueden (y deben servir) para varios grupos de estudiantes a la vez. Por tanto, ahorro de energía y esfuerzo por parte de los profesores a la hora de crear ejercicios y tutoriales.

b. Seguimiento y evolución uniforme de los estudiantes. Cuando los estudiantes tienen necesidades comunes, es interesante tener las mismas fuentes de información.

c. Mayor cantidad de información para el alumno. Cuando se da una buena distribución del trabajo, el esfuerzo del profesorado a la hora de crear handouts es menor porque los que se van creando pueden ser compartidos entre toda la comunidad de estudiantes. Además, algunos de estos trabajos pueden ser creados por los alumnos y simplemente recibir retroalimentación del profesor (Shih et al., 2008). 
Red U - Revista de Docencia Universitaria. Número Monográfico V. Número especial dedicado a WIKI y educación superior en España (II parte), en coedición con Revista de Educación a Distancia (RED). http://www.um.es/ead/Red_U/m5/

d. Ahorro económico en países en vías de desarrollo (la labor humanitaria y altruista) (Foster, 2008; Ravid et al., 2008). En países donde los libros de texto de calidad son excesivamente caros, las wikis pueden servir para proveer materiales adecuados a coste muy reducido.

Como afirma Robertson (2008, p. 437):

[...] wikis embed a set of characteristics that are consistent with adoption by teachers. That is, they can be perceived to provide a relative advantage over existing practices when applied to problem based and group based activities. They lack complexity, are flexible enough to be compatible with a range of teaching strategies, can be trialed easily and the results can be observed easily. Given that teachers are reasonably disposed to and aware of the possible use of technology in their practice, the technology may be adopted in the presence of a meaningful and authentic opportunity where there is perceived to be an adequate level of support.

Naturalmente, las funciones de los profesores están muy estrechamente relacionados con el tipo de alumno al que va dirigida la experiencia; es decir, con su interés, su capacidad lingüística, edad, autonomía, etc. pero, en general, hemos de destacar las siguientes funciones:

1) Crear un entorno adecuado al aprendizaje mediante la creación de una atmosfera creativa y solidaria presencial y virtual;

2) Realizar el control y moderación de la wiki. Es verdad que muchos profesores tratan (en nuestra opinión positivamente) de delegar algunas funciones de moderación en los alumnos para desarrollar su capacidad de autogestión y responsabilidad en el aprendizaje;

3) Proporcionar las condiciones en las que los alumnos entiendan la funcionalidad y su papel creativo en el desarrollo de la wiki;

4) Poner de relieve y explicitar lo que los alumnos tienen que hacer y cómo, así como distribuir las distintas asignaciones de control, mantenimiento y creación (o renovación) de la wiki;

5) Juzgar las aportaciones de los alumnos y, cuando corresponda, realizar la evaluación de las mismas;

6) Ayudar a los alumnos con sus posibles dificultades y dudas;

7) Animar a los alumnos a mejorar progresivamente. Esto no solamente se aplica a la lengua extranjera sino a las ideas, procurando guie al alumno hacia la excelencia;

8) Desarrollar unas relaciones positivas profesor-alumno y alumno-alumno;

9) Realizar las demostraciones necesarias del producto a desarrollar; $y$

10) Proveer modelos lingüísticos que faciliten el aprendizaje y el desarrollo de la wiki.

Este trabajo realizado por el profesor ayudaría a desmentir la idea tan generalizada de que lo que transmite el aprendizaje asistido por ordenador en general (Gudea, 2008), y muy especialmente a través de aplicaciones como las wikis, son fundamentalmente las "unidades de contenido" que parecen haber creado un conocimiento analítico en los estudiantes, distanciándoles de aprendizajes mediante procesamientos holísticos. Es decir, la redefinición del papel del profesor en el proceso 
Red U - Revista de Docencia Universitaria. Número Monográfico V. Número especial dedicado a WIKI y educación superior en España (II parte), en coedición con Revista de Educación a Distancia (RED). http://www.um.es/ead/Red_U/m5/

creativo de la wiki permite pasar de unidades de L2 y conocimientos (como el uso del pasado simple en una unidad temática como "historia de nuestro colegio" o "los músicos británicos de los 60") a procesamientos completos integrados (como "ayer y hoy de la música británica" desde la perspectiva audiovisual incluyendo léxico musical, social y sonidos de la época mediante la escritura y reescritura de un mismo texto [Mak y Coniam, 2008]). Por tanto, la wiki nos permite dejar atrás un aprendizaje instrumental para adentrarnos en las conexiones del entorno (como el constructivismo tradicional), el procesamiento personal (constructivismo social) y, además, centrarnos en el propio aprendizaje individualizado a través de la práctica continuada y monitorizada de la producción de la L2 por parte del alumno (cognitivismo). De esta manera, tanto el profesor como el alumno entran en un proceso de reflexión sobre la producción y la importancia de la modificación interna del conocimiento que tan de acuerdo está con la teoría del ínput (Krashen, 1982) y del output (Hanaoka, 2007).

\section{Las wikis para los alumnos}

La creación de wikis permite adquirir una conciencia y control de aprendizaje progresivamente a través de las siguientes facetas desarrolladas en cooperación con el profesor:

1) Sentido de la competencia. Los alumnos van adquiriendo conciencia de su propio aprendizaje al tener que desarrollar contenidos en los que su propia creación es fundamental. En este sentido, la interacción entre su producción y el control del profesor (a través de correcciones antes de la edición y publicación del output) son fundamentales en el aprendizaje.

2) Control del comportamiento lingüístico. El alumno usa las estructuras de manera repetida hasta adquirir una maestría en las mismas y finalmente incorporarlo a su producción no ritualizada (lo que denominaríamos incorrectamente "producción libre").

3) Fijación de objetivos. El alumno puede negociar los objetivos y contenidos con su profesor. Eso lo hace autónomo, pero también consciente de la importancia de la cooperación. Es decir, el aprendizaje del idioma le lleva a desarrollar sus capacidades de interacción social profesor-alumno y alumnoalumno. Además, puede gestionar y modificar sus aportaciones y objetivos específicos.

4) Consciencia del cambio. El alumno puede modificar sus propios objetivos, por lo que tiene que justificar su evolución y adquiere la conciencia de la importancia y la justificación del cambio.

5) Sentimiento positivo sobre los propios logros. Esta sensación evidentemente motivadora es muy importante tanto en el aprendizaje general como en el de una lengua extranjera.

A estos factores debemos añadir la importancia de la motivación interactiva (Williams y Burden, 1997), según la cual, los estudiantes involucrados en el proceso de compartir e incrementar sus concomimientos a través de la creación y el desarrollo de wikis ponen como foco de su trabajo el contexto social compartido con sus profesores y 
Red U - Revista de Docencia Universitaria. Número Monográfico V. Número especial dedicado a WIKI y educación superior en España (II parte), en coedición con Revista de Educación a Distancia (RED). http://www.um.es/ead/Red_U/m5/

sus alumnos y necesitan establecer sus razones para mejorar (y presentar unos contenidos adecuados en forma e ideas) a través de un esfuerzo que se encuentra justificado por una retroalimentación positiva del profesor (especialmente cuando admite los contenidos en la wiki) o, incluso, por la evaluación de sus compañeros (Lund, 2008b; Xiao y Lucking, 2008) y una voluntad de decisión importante. Como consecuencia el alumno quiere mejorar para sí mismo, para obtener la aprobación de su profesor y de sus compañeros, pudiéndose ampliar este círculo según lo abierta o cerrada que esté la wiki. Además, en nuestra opinión, la wiki permite cierto anonimato en el trabajo del alumno que puede desear ser cada vez mejor y triunfar sin que ello le suponga un cierto rechazo en su contexto social.

Independientemente de que el alumno, al practicar sus habilidades sociales, encuentre una motivación para su trabajo y de que el profesor le facilite la adquisición de la L2 a través de la interacción entre su producción y la retroalimentación recibida, puede seguir mejorando sus propias estrategias de aprendizaje tales como: fijarse en la forma y el significado ( $\sin$ disociar una de la otra), repetir palabras y estructuras prefijadas, monitorizar su propia producción, evaluarse a sí mismo a través de su grado de acierto-error (especialmente a la luz de las correcciones y modificaciones del profesor), crear sus propias reglas para el contexto de su entrada en la wiki, relacionar contenidos e ideas con la L2, planear su propio aprendizaje, revisar su producción, etc. (Kessler, 2009), estrategias éstas que son comúnmente usadas para mejorar la L2 de manera individualizada, aunque el alumno ha de trabajar otras basadas en la interacción con otros estudiantes (Figura 7).

\begin{tabular}{|l|l|}
\hline Tipo de estrategia & Estrategias usadas en el desarrollo y creación de wikis \\
\hline Estrategias cognitivas & $\begin{array}{l}\text { Practicar, recibir y enviar mensaje, razonar, revisar las } \\
\text { estructuras de input y output. }\end{array}$ \\
\hline $\begin{array}{l}\text { Estrategias de } \\
\text { compensación }\end{array}$ & Superar las propias limitaciones de escritura. \\
\hline Estrategias afectivas & $\begin{array}{l}\text { Automotivarse, reducir la ansiedad (claramente afectado } \\
\text { por el contexto cooperativo de Internet, autorregular la } \\
\text { temperatura emocinal. }\end{array}$ \\
\hline Estrategias sociales & Cooperar con otros, hacer preguntas, empatizar con otros. \\
\hline
\end{tabular}

Figura 7: Estrategias reguladas por la interacción de alumno en su contexto (basado en Oxford, 1990: 17).

\section{Conclusiones}

En este artículo hemos hecho un breve repaso a las características sociales de las wikis para a continuación referirnos a una teoría del conocimiento que tiene en cuenta la complementariedad de diversos estilos cognitivos; por una parte un aprendizaje más abstracto y, por otra, otro más experiencial, puesto que las wikis se relacionan con el primero. A continuación se ha hecho una breve descripción de dos de las metodologías docentes con las que se pueden asociar las actividades didácticas basadas en la construcción cooperativa de una wiki; por una parte, el método que se basa en la resolución de problemas y, por otra, el que tiene como objetivo desarrollar un proyecto entre varios compañeros. En el siguiente apartado nos hemos referido a la relación entre el estudiante de una lengua extranjera y la creación de una wiki, haciendo especial hincapié en el proceso cognitivo del estudiante que participa activamente en la 
Red U - Revista de Docencia Universitaria. Número Monográfico V. Número especial dedicado a WIKI y educación superior en España (II parte), en coedición con Revista de Educación a Distancia (RED). http://www.um.es/ead/Red_U/m5/

aportación escrita a una wiki. Por último, se le ha presentado al lector un breve listado de las ventajas del trabajo cooperativo docente en materia de wikis, para terminar con las relaciones que se establecen entre las wikis y el docente y éstas respecto al discente.

A modo de resumen, podemos concluir diciendo que, en nuestra opinión, las wikis son un excelente motor para el desarrollo de los aspectos más importantes del aprendizaje y la práctica de una lengua extranjera, no limitándose únicamente a ser tan sólo un contexto para el aprendizaje de esa L2, sino auténticos motores que integran tareas, funciones y colaboración entre los distintos agentes del aprendizaje (alumnos y profesores), además de ser un medio muy motivador (Oreg y Nov, 2008) para reforzar otras áreas educativas y mejorar el conocimiento general del mundo que tienen los alumnos. La gran ventaja es su utilidad y versatilidad que permite, tanto el trabajo colectivo como el individual.

Artículo concluido el 19 de noviembre de 2009

Gimeno, A. y García, J. (2009). Wikis y el nuevo estudiante de lenguas extranjeras. Red $U$ - Revista de Docencia Universitaria. Número Monográfico $V$. Número especial dedicado a WIKI y educación superior en España (II parte), en coedición con Revista de Educación a Distancia (RED). 31 de diciembre de 2009. Consultado el [dd/mm/aaaa] en http://www.um.es/ead/Red_U/m5/

\section{REFERENCIAS}

Balkcom, S. (1992) Cooperative Learning. Education Research Consumer Guide. Office of Educational Research and Improvement. U.S. Dept. of Education. Office of Research. Disponible el 12 de noviembre de 2009 en http://www.ed.gov/pubs/OR/ConsumerGuides/cooplear.html

Boule, M. (2008) Go with the flow: Selling social networking. School Library Journal, 54(11), 50-52.

Davidson, M. (2008) Using focus groups to learn about my wiki. Computers in Libraries, 28(1), 16-19, 47-48.

Engstrom, M. E., \& Jewett, D. (2005) Collaborative learning the wiki way. TechTrends: Linking Research \& Practice to Improve Learning, 49(6), 12-16. Disponible el 23 de agosto de 2009 en http://www.aect.org/Intranet/Publications/techtrends/4906.asp.

Forte, A. y Bruckman, A. (2006) From Wikipedia to the Classroom: Exploring Online Publication and Learning. Proceedings of the 7th international conference on Learning Sciences. Georgia: International Society of the Learning Sciences, pp 182 188. Disponible el 26 de agosto de 2009 en http://www.cc.gatech.edu/ asb/papers/forte-bruckman-icls06.pdf

Forte, A. y Bruckman, A. (2007) Constructing Text: Wiki as a Toolkit for (Collaborative?) Learning. Proceedings of the 2007 international symposium on Wikis. New York: ACM publications, pp. 31 - 42. 
Red U - Revista de Docencia Universitaria. Número Monográfico V. Número especial dedicado a WIKI y educación superior en España (II parte), en coedición con Revista de Educación a Distancia (RED). http://www.um.es/ead/Red_U/m5/

Foster, A. L. (2008) Providing online textbooks to the developing world. Education Digest: Essential Readings Condensed for Quick Review, 73(7), 14-16.

Galloway, C. M. (2001) Social Constructivism. En M. Orey (Ed.), Emerging perspectives on learning, teaching, and technology. Disponible el 8 de noviembre de 2009 en http://projects.coe.uga.edu/epltt/.

García Laborda, J. (2009) Using webquests for oral communication in English as a foreign language for Tourism studies. Educational technology and Society, 12(1), 258-270.

Gerlach, J. M. (1994) Is this collaboration? En Bosworth, K. y Hamilton, S. J. (Eds.), Collaborative Learning: Underlying Processes and Effective Techniques. New Directions for Teaching and Learning $N^{\circ} 59$.

Gudea, S. (2008) Expectations and demands in online teaching: Practical experiences. Hershey, PA: Information Science Publishing/IGI Global.

Hanaoka, O. (2007) Output, noticing, and learning: An investigation into the role of spontaneous attention to form in a four-stage writing task. Language Teaching Research, 11(4), 459-479.

Holec, H. (1997) Autonomie et apprentissage des langues étrangères. Autonomie \& enseignement. Apprentissage des langues étrangères. Ed. Bernard André. Paris: Didier.

Jones, D. (1996) What is Problem-Based Learning? Disponible el 10 de junio de 2009 en http://edweb.sdsu.edu/clrit/learningresource/PBL/WhatisPBL.html.

Kessler, G. (2009). Student-initiated attention to form in wiki-based collaborative writing. Language Learning \& Technology, 13(1), 79-95.

Kim, B. (2001) Social Constructivism. En M. Orey (Ed.), Emerging perspectives on learning, teaching, and technology. Disponible el 8 de noviembre de 2009 en http://projects.coe.uga.edu/epltt/.

Lund, A. (2008a) Wikis: A collective approach to language production. ReCALL, 20(1), $35-54$.

Lund, A. (2008b). Assessment made visible: Individual and collective practices. Mind, Culture, and Activity, 15(1), 32-51.

Mak, B., \& Coniam, D. (2008) Using wikis to enhance and develop writing skills among secondary school students in Hong Kong. System: An International Journal of Educational Technology and Applied Linguistics, 36(3), 437-455.

Morgan, B., \& Smith, R. D. (2008) A wiki for classroom writing. Reading Teacher, 62(1), 80-82.

Oreg, S. y Nov, O. (2008) Exploring motivations for contributing to open source initiatives: The roles of contribution context and personal values. Computers in Human Behavior, 24(5), 2055-2073.

Oxford, R. (1990) Language learning strategies: what every teacher should know. New York: Newbury House. 
Red U - Revista de Docencia Universitaria. Número Monográfico V. Número especial dedicado a WIKI y educación superior en España (II parte), en coedición con Revista de Educación a Distancia (RED). http://www.um.es/ead/Red_U/m5/

Papert, S. (1991) Situating Constructionism. En Harel, I. y Papert, S. (Eds.), Constructionism, Norwood, NJ: Ablex Publishing.

Ravid, G., Kalman, y Rafaeli, S. (2008) Wikibooks in higher education: Empowerment through online distributed collaboration: Internet empowerment. Computers in Human Behavior, 24(5), 1913-1928.

Robertson, I. (2008) Learners' attitudes to wiki technology in problem based, blended learning for vocational teacher education. Australasian Journal of Educational Technology, 24(4), 425-441. Disponible el 8 de noviembre de 2009 en http://www.ascilite.org.au/ajet/ajet24/robertson.html

Royal, C. \& Kapila, D. (2009) What's on wikipedia, and what's not...?: Assessing completeness of information. Social Science Computer Review, 27(1), 138-148.

Schneider, D.K. (2006) EduTech Wiki. Disponible el 8 de noviembre de 2009 en http://edutechwiki.unige.ch/en/Main_Page.

Schrock, K. (2007) Critical evaluation in the collaborative era: What role should a wiki play in authentic research? Technology \& Learning, 28(3), 38.

Shih, W., Tseng, S., \& Yang, C. (2008) Wiki-based rapid prototyping for teachingmaterial design in E-learning grids. Computers \& Education, 51(3), 1037-1057.

Stacey, P. (2008) 'Wikivism': From communicative capitalism to organized networks. Cultural Politics, 4(1), 73-99.

Toker, S., Moseley, J. L., \& Chow, A. T. (2008) Is there a wiki in your future?: Applications for education, instructional design, and general use. Educational Technology Magazine: The Magazine for Managers of Change in Education, 48(5), 22-27.

Vygotsky, L.S. (1962) Thought and Language. Cambridge, MA: MIT Press.

Wheeler, S., Yeomans, P., \& Wheeler, D. (2008) The good, the bad and the wiki: Evaluating student-generated content for collaborative learning. British Journal of Educational Technology, 39(6), 987-995.

Williams y Burden (1997) Psychology for language teachers: A social constructivist approach. New York: Cambridge University Press.

Xiao, Y., \& Lucking, R. (2008) The impact of two types of peer assessment on students' performance and satisfaction within a wiki environment. Internet and Higher Education, 11(3-4), 186-193.

Zorko, V. (2007) A rationale for introducing a wiki and a blog in a blended-learning context. CALL-EJ Online, 8(2), Disponible el 8 de noviembre de 2009 en http://www.tell.is.ritsumei.ac.jp/callejonline/journal/8-2/zorko.html.

\footnotetext{
'http://en.wikipedia.org/wiki/Ward_Cunningham (Fecha de último acceso: 08/11/2009)

ii http://www.colegiosanagustin.net/wiki/index.php?title=Portada (Fecha de último acceso: $05 / 11 / 2009)$

iii http://teatro.webs.upv.es/ (Fecha de último acceso: 05/11/2009)

${ }^{i v} \mathrm{http}: / /$ wiki.eflgeek.com (Fecha de último acceso: 08/11/2009)

${ }^{\vee}$ Para más información, véase el vídeo en

http://aulablog21. wikispaces.com/TALLER+DE+WIKIS (Fecha de último acceso: 08/11/2009)
} 
Red U - Revista de Docencia Universitaria. Número Monográfico V. Número especial dedicado a WIKI y educación superior en España (II parte), en coedición con Revista de Educación a Distancia (RED). http://www.um.es/ead/Red_U/m5/

${ }^{v i}$ Payton, T. (2004) Collaborative Projects, en Payton Educational Consulting

http://www.tammypayton.net/courses/collab/what.shtml (Fecha de último acceso: 08/11/2009)

vii Aprendizaje profundo en contraposición al aprendizaje superficial. Weigel, V. B. (2002). Deep learning for a digital age: Technology's untapped potential to enrich higher education. San Francisco: Jossey-Bass.

viii Del inglés CLIL, acrónimo de Content and Language Integrated Learning.

${ }^{i x}$ http://edutechwiki.unige.ch/en/Main_Page. (Fecha de último acceso: 08/11/2009) 